\title{
Prescrição de medicamentos: uma análise para a implantação da prescrição eletrônica ambulatorial
}

\author{
Prescription of drugs: an analysis for implantation of outpatient electronic prescription \\ Prescripción de medicamentos: un análisis para la implementación de prescripción \\ electrónica ambulatoria
}

Luís Henrique Oliveira Rezende ${ }^{1 *}$, Flávia de Sousa Gehrke ${ }^{1}$, Michele Aparecida Silva ${ }^{1}$, Alexandre Mansuê Ferreira Carneiro², Rodrigo Martins $\mathrm{Abreu}^{3}$, Camila Nascimento Monteiro ${ }^{4}$, Kemile Albuquerque Leão ${ }^{5}$, Kioko Takei ${ }^{1}$.

\section{RESUMO}

Objetivo: Analisar as não conformidades nas receitas recebidas na farmácia ambulatorial de um hospital estadual de São Paulo, sendo a base para a proposta de implantação do módulo de prescrição eletrônica. Métodos: Trata-se de estudo descritivo, com a análise das principais formas de elaboração de receitas atendidas entre 01 a 30 de março de 2018. Utilizando para isso, um roteiro com variáveis: Tipo de receita; Caligrafia ou grafia; Uso de abreviaturas; Utilização da DCB; Especificação do tratamento. Resultados: Das receitas analisadas (96\%) apresentaram erros. Das manuscritas (16\%) com erro de redação/caligrafia, (13\%) sem identificação do prescritor, (93\%) apresentaram abreviaturas e $(29 \%)$ não traziam a DCB. Nas digitadas, (97\%) apresentaram erros, em (76\%) presença de abreviaturas e em $(24 \%)$ ausência da DCB. Os erros de decisão nas manuscritas, a ausência de potência em (7\%) e (79\%) não mostravam a quantidade de medicamentos a serem dispensadas e (17\%) sem a duração do tratamento. Nas digitadas (74\%) não apresentaram a quantidade de medicamento a ser dispensada e em (37\%) ausência da duração do tratamento. Conclusão: A implantação da prescrição eletrônica ambulatorial pode significar a redução de fatores que induzem a erros e ausência de informações inerentes aos esquemas farmacoterapêuticos prescrito para o paciente.

Palavras-chave: Prescrições de medicamentos, Erros de medicação, Uso de medicamentos.

\section{ABSTRACT}

Objective: To analyze the non-conformities in the prescriptions received at the outpatient pharmacy of a state hospital in São Paulo, being the basis for the proposal to implement the electronic prescription module. Methods: This is a descriptive study, with the analysis of the main forms of preparation revenue served between March 1 to 30, 2018. Using this, a script with variables: Type of revenue; Calligraphy or spelling; Use of abbreviations; Use of DCB; Treatment specification. Results: Of the analyzed recipes (96\%) presented errors. Of the manuscripts (16\%) with writing/handwriting errors, (13\%) without identification of the prescriber, (93\%) had abbreviations and (29\%) did not have the DCB. In those typed, (97\%) had errors, $(76 \%)$ the presence of abbreviations and $(24 \%)$ the absence of DCB. The decision errors in the manuscripts, the lack of potency in (7\%), and $(79 \%)$ did not show the number of drugs to be dispensed and $(17 \%)$ without the duration of the treatment. In those typed (74\%) they did not show the amount of medication to be dispensed and in (37\%) the duration of treatment was absent. Conclusion: The implantation of an outpatient electronic prescription can mean the reduction of factors that induce errors and the absence of information inherent to the pharmacotherapeutic schemes prescribed for the patient.

Key words: Drug prescriptions, Medication errors, Use of medicines.

\section{RESUMEN}

Objetivo: Analizar las no conformidades en las recetas recibidas en la farmacia ambulatoria de un hospital estatal de São Paulo, siendo la base de la propuesta para implementar el módulo de prescripción electrónica. Métodos: Estudio descriptivo, con el análisis de las principales formas de preparación. ingresos atendidos entre el 1 y el 30 de marzo de 2018. Utilizando esto, un script con variables: tipo de ingresos; Caligrafía u ortografía; Uso de abreviaturas; Uso de DCB; Especificación de tratamiento. Resultados: De las recetas analizadas (96\%) se presentaron errores. De los manuscritos $(16 \%)$ con errores de escritura / escritura a mano, (13\%) sin identificación del prescriptor, (93\%) tenían abreviaturas y $(29 \%)$ no tenían el DCB. En los tipificados, $(97 \%)$ tenían errores, $(76 \%)$ la presencia de abreviaturas y $(24 \%)$ la ausencia de DCB. Los errores de decisión en los manuscritos, la falta de potencia en (7\%) y (79\%) no mostraron la cantidad de

\footnotetext{
${ }^{1}$ Instituto de Assistência Médica ao Servidor Público Estadual (IAMSPE), São Paulo - SP. *E-mail: rezendehluis@yahoo.com.br ${ }^{2}$ Centro Universitário do Estado do Pará (CESUPA), Belém - PA.

${ }^{3}$ Universidade de São Paulo (USP), São Paulo - SP.

${ }^{4}$ Hospital Israelita Albert Einstein, São Paulo - SP.

${ }^{5}$ Faculdade Dinâmica do Vale do Piranga, Ponte Nova - MG.
} 
medicamentos a dispensar y (17\%) sin la duración del tratamiento. En los tipados (74\%) no mostraron la cantidad de medicación a dispensar y en (37\%) la duración del tratamiento estuvo ausente. Conclusión: La implantación de prescripción electrónica ambulatoria puede significar la reducción de factores que inducen errores y ausencia de información inherente a los esquemas farmacoterapéuticos prescritos para el paciente.

Palabras clave: Recetas de medicamentos, Errores de medicación, Uso de medicamentos.

\section{INTRODUÇÃO}

A prescrição médica é classificada como um documento clínico de valor legal, tornando-se responsabilidade daquele que prescreve, manipula, dispensa e administra a terapêutica, seja ela considerada medicamentosa ou não. Constituído por normas gerais, um dos seus principais objetivos é o fornecimento de instruções claras ao paciente e demais profissionais da saúde, referentes à terapêutica, garantindo a interpretação e a objetividade das informações prestadas (OSORIO-DE-CASTRO CGS e PEPE VLE, 2012). Em âmbito nacional e internacional apresentam-se leis que precedem a elaboração de uma prescrição de medicamentos e abrangem aspectos éticos que devem ser obedecidos pelos prescritores. As principais leis envolvidas na elaboração de prescrições de medicamentos no Brasil são: as Leis Federais 5.991/73 e 9.787/99, as Resoluções 1.552/99, 1.477/97, 1.885/2008 do Conselho Federal de Medicina (CFM) e a Resolução 357/2001 do Conselho Federal de Farmácia (CFF) (OSORIO-DE-CASTRO CGS e PEPE VLE, 2012).

No âmbito do Sistema Único de Saúde (SUS), a prescrição deverá adotar a Denominação Comum Brasileira (DCB). O documento não deve conter abreviaturas (OSORIO-DE-CASTRO CGS e PEPE VLE, 2012). O uso da DCB, além de obrigatório no âmbito do SUS, reduz as confusões entre nomes de medicamentos. Por outro lado, a utilização da denominação comercial pode induzir a erros, devido às variações dos nomes comerciais de um país para outro que estão sujeitos a mudanças para atender aos interesses de mercado (GUZATTO P e BUENO D, 2007). Ainda neste mesmo limiar de discussão, temos o Decreto $n^{\circ}$ 20.931/32 que regulamenta que a prescrição deve ser escrita por extenso e de forma clara, sendo vedado ao médico prescrever de forma secreta, através do uso de códigos (símbolos).

As legislações vigentes apontam ainda que as prescrições médicas devam ser legíveis, sem a presença de rasuras, datadas e assinadas com clareza, de modo a garantir a comunicação efetiva entre prescritor, paciente e os outros profissionais de saúde, em especial farmacêutico e enfermeiro. Obedecer a esses quesitos possibilita a detecção de possíveis erros por parte de outros profissionais de saúde, antes do início da terapia medicamentosa (EV LS, et al., 2008). Erros na administração de medicamentos podem ocorrer, muitas vezes, em etapas do processo que dependem exclusivamente da ação humana. Contudo, condições que auxiliem na prevenção e minimização desses erros, podem ser alcançadas através de um sistema de medicações bem estruturado, com normas, regras e processos bem implementados que podem auxiliar as ações dos profissionais de saúde envolvidos (EV LS, et al., 2008).

Dentro deste contexto, a Tecnologia da Informação (TI) vem sendo utilizada com o intuito de prevenir e/ou reduzir os erros de medicação. Neste sentido, tanto o uso do código de barras nas embalagens dos medicamentos, bem como a prescrição médica eletrônica são alguns dos mecanismos utilizados. Temos a prescrição médica como aquela onde o profissional médico (ou transcritor) utiliza um computador para registrar as informações da prescrição, utilizando para isso um modelo padronizado. Esse modelo tem a capacidade de reduzir os erros provocados por uma escrita ilegível e também a possibilidade de corrigir um erro de digitação durante a elaboração da prescrição, sem precisar rasurar ou rabiscar o documento, situação que dificulta mais ainda o entendimento e/ou interpretação das informações (SHANE R, 2002)

Analisar a qualidade da prescrição médica é avaliar uma parte dos serviços de saúde prestados à sociedade. A prescrição é também um documento que pode indicar a satisfação do paciente e sua adesão ao tratamento (GUZATTO P e BUENO D, 2007). Faz parte da adesão ao tratamento o entendimento da mesma, tanto pelos demais profissionais da saúde relacionados ao medicamento como pelo paciente. Se esses apresentarem dúvidas em relação ao que foi prescrito, erros de medicação (EM) podem ocorrer como também desmotivação ao tratamento e alterações nos objetivos a serem alcançados (CARVALHO VT, et al., 1999). 
Neste sentido, objetivou-se analisar as não conformidades nas receitas recebidas na farmácia ambulatorial, através da observação das principais formas de elaboração utilizadas em um Hospital no estado de São Paulo. Tal análise servirá de base para a elaboração da proposta de implantação da prescrição eletrônica, no sistema informatizado de gestão hospitalar já existente, utilizado para atendimento dos pacientes ambulatoriais.

\section{MÉTODOS}

Trata-se de estudo descritivo, baseado na análise de dados extraídos das receitas de medicamentos atendidas no período de 1 a 30 de março de 2018na farmácia ambulatorial de um Hospital Estadual, caracterizado como um hospital geral, de nível quaternário e de ensino, localizado na capital de São Paulo. A farmácia ambulatorial realiza em média 19.000 dispensações por mês. Levou-se em consideração este número para a determinação da amostra, utilizando-se para isso a calculadora on-line. Com nível de confiança de $95 \%$ e erro amostral de 5\%, a amostra mínima necessária foi de 377 receitas. Contudo, analisou-se um total de 3.506 receitas médicas.

A coleta de dados foi realizada por conveniência de forma aleatória, obtida através de cópia digital das receitas atendidas, no período de atendimento entre 9:00 às 11:00 e de 14:00 às 16:00 (de segunda a sextafeira), sendo armazenadas e categorizadas por números em um banco de dados eletrônico. Para tal, foi criado um roteiro analítico embasado nos marcos regulatórios nacionais: leis 5.991/73 e 9.787/99. E nos critérios de boas práticas de prescrição de medicamentos citados nas Resoluções 1.552/99, 1.477/97, 1.885/2008 do Conselho Federal de Medicina (CFM) e a Resolução 357/2001 do Conselho Federal de Farmácia (CFF).

O roteiro foi composto considerando as seguintes variáveis: Tipo de receita, classificadas como: Digitada - aquelas confeccionadas utilizando o sistema informatizado MVSOUL $\circledast$ existente no serviço ambulatorial do hospital, que disponibiliza uma ferramenta para digitação da prescrição dentro do módulo de atendimento ao paciente ambulatorial. Através desta, o prescritor pode optar por realizar a prescrição na forma digitada, acessando o campo de livre digitação, destinado a prescrição dos medicamentos. Já a Manuscrita - aquela que apresenta qualquer tipo de informação do paciente, medicamento e/ou tratamento escrita a próprio punho. Caligrafia ou grafia - classificada como ilegível quando pelo menos uma palavra não foi identificada. Analisando também a presença e ausência das seguintes variáveis: Nome do paciente; Data da prescrição; Identificação do prescritor; Uso de abreviaturas; Utilização da DCB; Especificação do medicamento; Potência do medicamento; Quantidade de medicamento a ser dispensada; Posologia e Duração do tratamento.

Um erro de prescrição clinicamente significativo ocorre quando - como resultado de uma decisão ou no processo de redação da prescrição - há uma redução significativa, não intencional, na probabilidade de o tratamento ser efetivo ou ocorre um aumento do risco de dano, se comparado com a prática geralmente aceita (DEAN B, et al., 2000). Desta forma, os erros de prescrição foram divididos em: erros de decisão e erros de redação. Sendo erros de decisão as seguintes variáveis: especificação do medicamento, potência do medicamento, quantidade de medicamento a ser dispensada, posologia, duração do tratamento. Já os erros de redação: caligrafia (ilegibilidade), identificação do paciente e do prescritor, data, não utilização da DCB e abreviaturas.

Os dados de legibilidade da prescrição foram analisados pelo autor desta pesquisa e por mais um farmacêutico especializado no atendimento das receitas ambulatoriais. Nos casos de discordância, um terceiro profissional farmacêutico, também com experiência no atendimento ambulatorial foi consultado, a fim de se estabelecer um consenso sobre a ilegibilidade ou não da prescrição. Foi utilizada a classificação de Rosa MB, et al. (2003), que preconiza que cada palavra deve ser examinada e avaliada separadamente com o intuito de evitar a interpretação ou a dedução. A autora estabelece, portanto, que a prescrição deve ser entendida e não interpretada.

Foram excluídas do estudo receitas atendidas fora do período e do horário de atendimento estabelecido. Ressalta-se que o período escolhido de um mês, se justifica pelo fato de que a maioria dos medicamentos dispensados nessa farmácia é de uso contínuo. Logo, as receitas têm validades variadas, de um a 12 meses, o que levaria a uma duplicação das receitas a serem analisadas. Foram excluídas também as receitas referentes aos medicamentos do Componente Especializado da Assistência Farmacêutica. Tais medicamentos contam com regulamento próprio através da Portaria ํㅜ⒈554, de 30 de julho de 2013. 
Os dados foram digitados em planilhas do programa Microsoft Excel 2013 (Microsoft Corporation, Redmond, WA, Estados Unidos), trabalhados em sua consistência, revistos e analisados. A análise de associação dos dados foi feita por meio dos testes de Qui-quadrado e Teste exato de Fisher para verificação da associação entre o tipo de receita e os erros. O estudo atendeu às normas e diretrizes do CEP, sendo aprovado sob número de parecer 1.498.521.

\section{RESULTADOS}

Das 3.506 receitas que compuseram a amostra, 3.372 (96\%) apresentaram erros. Dentre elas, $2.825(81 \%)$ foram classificadas como manuscritas e $681(19 \%)$ digitadas. Nas manuscritas, $2.713(96 \%)$ apresentaram erros: 394 receitas (15\%) com um erro, $1.178(43 \%)$ com dois erros e $1.244(46 \%)$ com três ou mais erros. Dentre as digitadas, das 681 receitas, 659 (97\%) apresentaram erros: $179(27 \%)$ um erro, $290(44 \%)$ dois erros e 190 (29\%) 3 ou mais erros (Figura 1).

Figura 1 - Organograma: composição da amostra.

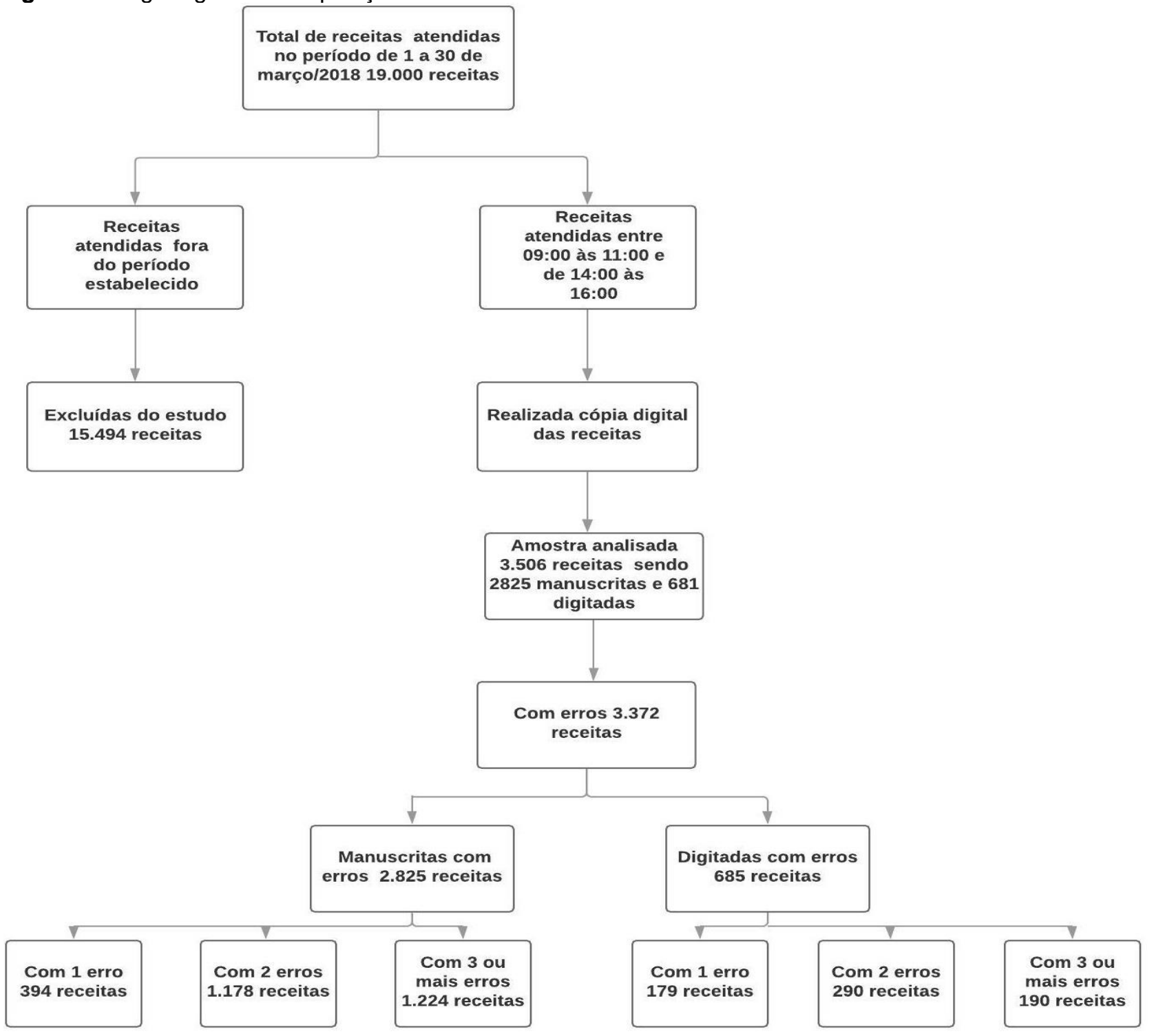

Fonte: Rezende LHO, et al, 2018.

Em relação aos erros de redação, dentre as 2.825 receitas analisadas, observou-se o uso de abreviatura em $2.536(93 \%)$ delas e apenas 35 (1\%) não apresentaram a identificação do prescritor. Daquelas digitadas $500 / 659$ (76\%) utilizaram abreviaturas e 157/659 (24\%) não utilizaram a DCB (Tabela 1). 
Tabela 1 - Distribuição dos erros de redação encontrados nas receitas manuscritas e digitadas

\begin{tabular}{cccc}
\hline Erros de redação & Manuscritas $(\mathbf{n}) \%$ & Digitadas $(\mathbf{n}) \%$ & Valor p \\
\hline Caligrafia (legibilidade) & $\mathbf{n}=\mathbf{2 . 7 1 3}$ & $\mathbf{n = 6 5 9}$ & \\
Ausência da data da prescrição & $446 / 2.713(16 \%)$ & $0 / 659$ & $<0,0001$ \\
Ausência da Identificação do prescritor & $747 / 2.713(28 \%)$ & $0 / 659$ & $<0,0001$ \\
Uso de abreviaturas & $35 / 2.713(1 \%)$ & $0 / 659$ & 0,0057 \\
Nãoutilização da DCB & $\mathbf{2 . 5 3 6 / 2 7 1 3 ( 9 3 \% )}$ & $\mathbf{5 0 0 / 6 5 9 ( 7 6 \% )}$ & $<0,001$ \\
& $773 / 2713(29 \%)$ & $157 / 659(24 \%)$ & 0,0184 \\
\hline
\end{tabular}

Fonte: Rezende LHO, et al., 2018.

Os erros de caligrafia (ilegibilidade), ausência da data de prescrição e identificação do prescritor não foram encontrados nas receitas digitadas. Porém, o uso de abreviaturas foi encontrado em 500/659 (76\%) receitas, e a não utilização da DCB em 157/659 (24\%) das receitas com erros avaliadas (Figura 2).

Figura 2 - Exemplo de receita digitada e manuscrita, respectivamente, com a presença do erro de redação uso de abreviaturas

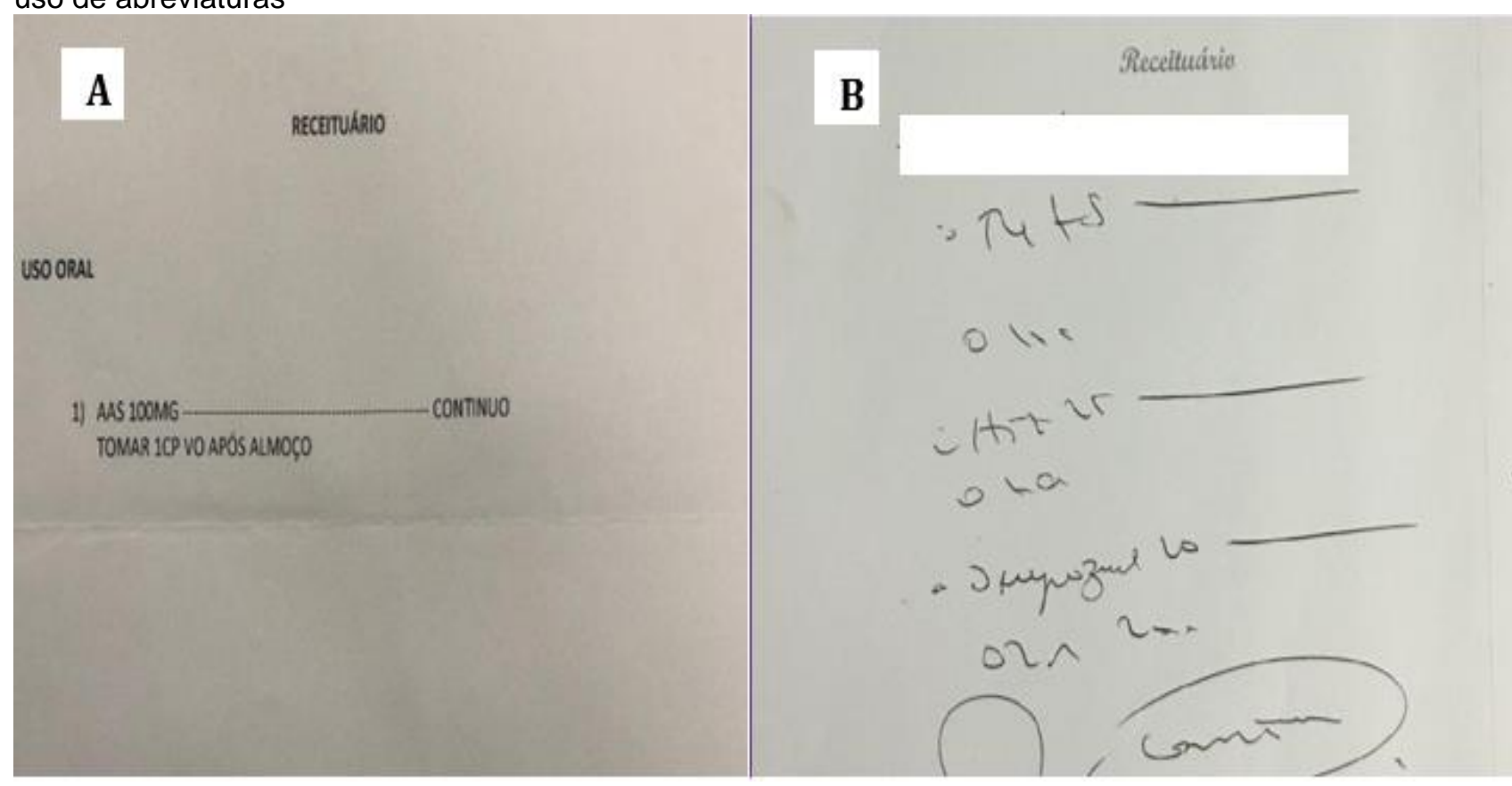

Legenda: (A) Receita digitada com uso de abreviatura no nome do medicamento, via de administração e na posologia. (B) Receita manuscrita com utilização de abreviaturas no nome do medicamento e na posologia. Fonte: Rezende LHO, et al., 2018.

No que se refere aos erros de decisão, $2.141 / 2.713$ (79\%) não apresentaram a quantidade de medicamentos a serem dispensados para o tratamento prescrito, resultado semelhante também foi encontrado nas receitas digitadas, onde 489/659 (74\%), apresentaram o mesmo erro, já a duração do tratamento foi ausente em maior número nesse tipo de receita com 246/659 (37\%) delas em comparação as manuscritas 453/2.713 (17\%) (Tabela 2).

Tabela 2 - Distribuição dos erros de decisão encontrados nas receitas manuscritas e digitadas.

\begin{tabular}{|c|c|c|c|}
\hline Erros de decisão & Manuscritas (n)\% & Digitadas (n)\% & Valor $p$ \\
\hline Especificacão do medicamento & $\begin{array}{c}\mathbf{n}=\mathbf{2 . 7 1 3} \\
130 / 2.713(5 \%)\end{array}$ & $\begin{array}{c}\mathbf{n}=\mathbf{6 5 9} \\
12 / 659(2 \%)\end{array}$ & 0.0005 \\
\hline Potência do medicamento & $181 / 2.713(7 \%)$ & $33 / 659(5 \%)$ & 0,0924 \\
\hline Quantidade de medicamentos a ser dispensado & $2.141 / 2.713(79 \%)$ & $489 / 659(74 \%)$ & 0,0103 \\
\hline Ausência de posologia & $64 / 2.713(2 \%)$ & $0 / 659$ & 0,0001 \\
\hline Duração do tratamento & $453 / 2.713(17 \%)$ & $246 / 659(37 \%)$ & $<0,0001$ \\
\hline
\end{tabular}

Fonte: Rezende LHO, et al., 2018. 
Figura 3 - Exemplo de receita manuscrita e digitada com a presença do erro de decisão ausência da quantidade de medicamento a ser dispensado.

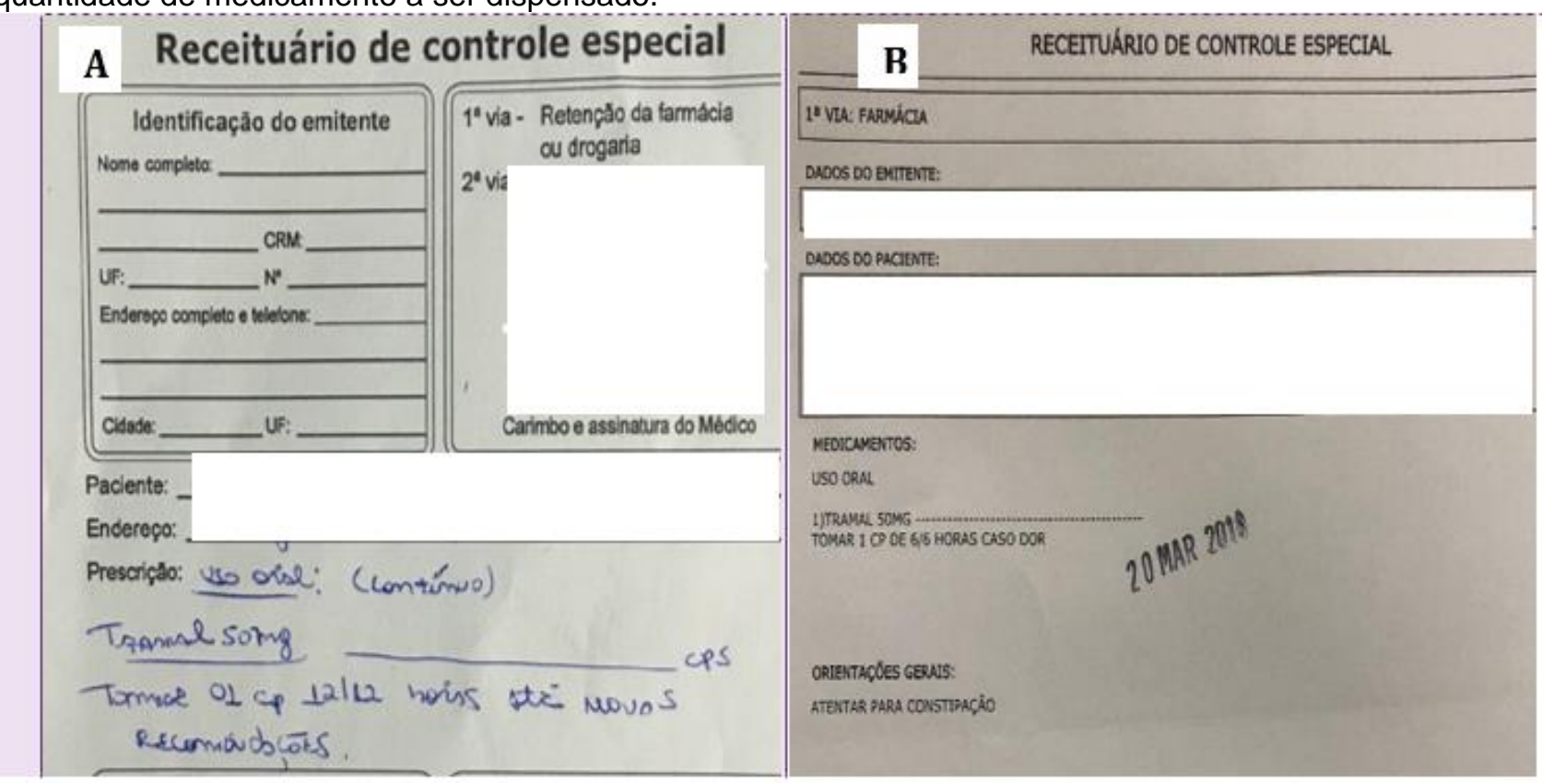

Legenda: (A) Receita manuscrita com a ausência da quantidade de medicamentos a ser dispensado. (B) Receita digitada com a ausência da quantidade de medicamentos a ser dispensado.

Fonte: Rezende LHO, et al., 2018.

\section{DISCUSSÃO}

No Brasil, milhões de receitas geradas no sistema públicos de saúde não apresentam os requisitos técnicos e legais imprescindíveis para uma dispensação eficiente e uso racional de medicamentos. Em um estudo que analisou 1.227 receitas, em uma unidade de atenção primaria à saúde em Aracaju, mostra que os dados apresentados além de preocupantes no que se refere aos agravos à saúde da população, também geram reflexos tanto nos recursos governamentais destinados à saúde, como também à própria sociedade (OTERO MJ, 2004).

No contexto da assistência farmacêutica, destaca-se o recebimento, a compreensão e a correta dispensação da receita médica um papel importante do profissional farmacêutico. Neste estudo, das 3.506 receitas analisadas 2.825 (81\%) eram manuscritas, corroborando com o estudo de Néri EDR (2004) realizado em um hospital universitário no Ceará, que também observou um número maior de receitas manuscritas sendo $440(72,15 \%)$, daquelas que foram analisadas, diferindo dos resultados encontrados porJacobsen TF, et al. (2015) na região sul do Brasil, que observou um número menor de receitas manuscritas sendo 860 $(30 \%)$ das receitas analisadas. Contudo, cabe ressaltar que existe certa carência de estudos que analisem as não conformidades em receitas destinadas ao paciente ambulatorial em âmbito hospitalar, pois a grande maioria dos estudos analisa a qualidade das prescrições voltadas para o paciente internado.

Entendemos que na farmácia ambulatorial do hospital estudado, a ilegibilidade da prescrição torna o processo de transcrição para o sistema eletrônico de dispensação um dos principais fatores de erros de dispensação ou retorno do paciente ao ambulatório para esclarecimento com o prescritor sobre os medicamentos prescritos. Problema agravado com a ausência dos dados do prescritor. Indo contra o que é preconizado na legislação brasileira, por exemplo, em seu artigo 35 da Lei n 5991/73 apontando que "será aviada a receita que estiver escrita à tinta. Em vernáculo por extenso e de modo legível, observados a nomenclatura e o sistema de medidas atuais". Entende-se que a presença da assinatura e carimbo, além de serem exigências legais, constituem uma possibilidade concreta de responsabilizar o prescritor e identificá-lo em caso de dúvida ou problema.

Outro erro de redação encontrado refere-se à ausência de data nas prescrições manuscritas, observado em $747(28 \%)$ das receitas avaliadas. Apesar de inicialmente parecer uma informação considerada 
dispensável, torna-se de grande importância, quando precisamos calcular o período de validade da receita. Uma vez que, no hospital estudado, prescrições de medicamentos de uso contínuo, que não aqueles da portaria 344 (que aprova o Regulamento Técnico sobre substâncias e medicamentos sujeitos a controle especial) têm validade de doze meses a partir da data da realização da prescrição médica.

O erro de redação "não utilização da DCB" e o uso de abreviaturas foram encontrados em ambos os tipos de receitas avaliadas neste estudo. Resultados que corroboram com os de Alves TNP, et al. (2012), quando analisou receitas médicas em unidades de atenção primária do município de Juiz de Fora-MG observando que as receitas manuscritas, $255(20 \%)$ eram redigidas com o nome comercial. Porém, Araújo PT-DE-B, (2011), em seu estudo observou o contrário, ao avaliar a qualidade das receitas de medicamentos de um hospital de ensino, onde das $1.509(49,81)$ receitas continham medicamentos prescritos pelo nome comercial.

O uso de abreviaturas está entre as causas mais citadas de erros de medicação por seu potencial de confusão e falhas de comunicação, seu uso foi proibido por legislação especifica (Decreto $n^{\circ} 20.931$ de 11 de janeiro de 1932, Revigorado pelo Decreto de 12 de julho de 1991, que regula e fiscaliza o exercício da medicina, da odontologia, da medicina veterinária e das profissões de farmacêutico, parteira e enfermeira, no Brasil, e estabelece penas). Jacobsen TF, et al. (2015), também observou em seu estudo, que as abreviaturas tanto do nome de medicamentos, quanto nas formas farmacêuticas, via de administração e posologia estavam presentes em $1.888(70,3 \%)$ das 2.678 receitas avaliadas. Diferente do resultado do estudo na farmácia ambulatorial realizado em Porto Alegre - RS, que analisou 3.701 receitas, onde foi observado que $233(6,3 \%)$ continham abreviaturas de nomes de medicamentos (GUZZATO P e BUENO D, 2007).

As abreviaturas utilizadas nas prescrições podem levar a várias interpretações, pois estas muitas vezes apresentam vários significados, fato que se torna mais grave quando o leitor não está familiarizado com seu significado, ou quando mal escrita certamente será confundida com outra abreviatura. Neri EDR (2004), aponta que o uso de abreviaturas de Unidades Internacionais (UI) foi associado a erros potencialmente fatais ou severos, observado em prescrições manuscritas de insulina, o "U" parecia com o "zero". Erro que pode multiplicar a administração de doses dez vezes maior do que a prescrita. Luiza VL e Gonçalves CBC (2009), ressaltam que não se devem usar abreviaturas para designar formas farmacêuticas, como por exemplo: comp. ou cap. ao invés de comprimido ou cápsula, respectivamente; vias de administração, VO ou IV, em vez de via oral ou via intravenosa; quantidades, 1 cx no lugar de "uma caixa"; ou intervalo entre doses, SN em vez de " se necessário" ou $2 / 2 \mathrm{~h}$, em vez de "a cada duas horas".

No estudo realizado por Alves TNP, et al. (2012), o erro de decisão ausência da quantidade de medicamentos a ser dispensada foi encontrado em 749 (58,8\%) das receitas manuscritas analisadas, corroborando com os resultados encontrados no presente estudo onde a ausência da quantidade de medicamento a ser dispensada foi encontrado em 2.142 (79\%) das receitas, sendo o erro mais encontrado, não somente nas receitas manuscritas como também nas digitadas, em um total de 489 (74\%). Segundo Rosa MB, et al. (2009), a diminuição dos erros de prescrição pode ocorrer com a implantação da prescrição eletrônica. Contudo, se faz necessário uma elaboração prudente daquelas prescrições pré-digitadas, bem como a utilização de ferramentas, como os editores de texto para elaboração da prescrição. Evitando possíveis novos tipos de erros, ou até mesmo a continuação de erros anteriores em um novo modelo de elaboração de prescrição.

Foi possível perceber que mesmo com a existência de uma ferramenta hospedada no sistema informatizado MVSOUL ${ }^{\circledR}$, para a digitação das prescrições geradas nos atendimentos aos pacientes ambulatoriais no hospital, o problema das não conformidades apresentadas nas receitas não foi solucionado. Os únicos erros que podem ter sido corrigidos com a utilização desta ferramenta referem-se aos erros de redação como aqueles relacionados à ilegibilidade, a data da prescrição e nome do médico prescritor, pois estes últimos são importados automaticamente para o cabeçalho da receita. Como nesta ferramenta o campo destinado a prescrição de medicamentos é apresentado de forma livre para digitação, o erro de redação uso de abreviaturas foi encontrado em frequência significativa, 500 (74\%), seguido da não utilização da DCB, encontrado em 157 (24\%). No que tange os erros de decisão, o único erro não encontrado foi a ausência da posologia, mostrando que uma ferramenta eletrônica sem parâmetros, com campo livre para digitação do prescritor não foi efetivo para eliminação dos erros que comprometem a qualidade das prescrições. 
Os resultados encontrados neste estudo foram apresentados para a diretoria clínica do hospital estudado, que perceberam a necessidade da elaboração de um módulo para prescrição eletrônica ambulatorial, dentro do sistema de gestão hospitalar MVSOUL ${ }^{\circledR}$, visando sanar os problemas de inconformidades presentes nas prescrições recebidas na farmácia ambulatorial do hospital, com o intuito de diminuir também a necessidade da transcrição das prescrições para o sistema de dispensação usado para atendimento aos pacientes.

A extinção da etapa de transcrição das prescrições evita a propagação dos erros, devido ao não entendimento das informações contidas nas prescrições. Promove também maior agilidade nos atendimentos e, consequentemente, diminuição do tempo de espera para retirada de medicamentos na farmácia, outro grande problema que muitas vezes afeta a continuidade dos tratamentos ou a adesão dos pacientes à terapia medicamentosa proposta. Resolvendo assim as inconformidades encontradas na atual ferramenta disponibilizada para digitação da prescrição e tentar gradualmente eliminar as prescrições manuscritas nos ambulatórios, pois poderíamos encontrar barreiras, quando um ou outro prescritor se recusasse a utilizar esta ferramenta.

Após ajustes necessários e através de constantes reuniões entre diretoria médica, diretoria técnica de farmácia e equipe de tecnologia da informação, foi proposto um módulo de prescrição eletrônica ambulatorial a ser disponibilizado no sistema MVSOUL®, sistema de gestão hospitalar já existente no hospital para atendimento ambulatorial (Figura 4). Ressalta-se aqui a importância de estudos que objetive avaliar a utilização e efetividade do modulo proposto após sua implantação.

Figura 4 -Tela teste para realização da prescrição eletrônica ambulatorial.

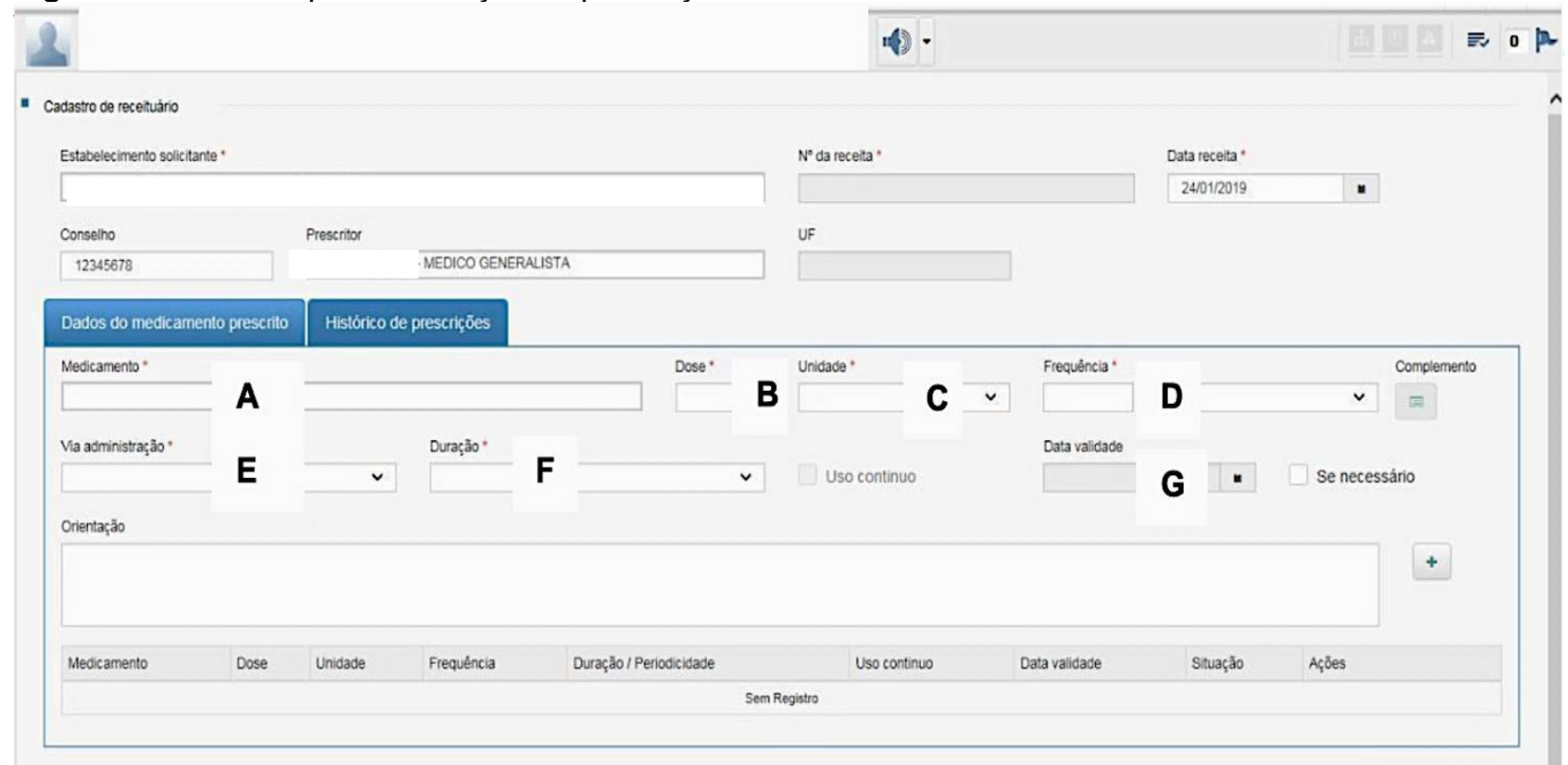

四早

Legenda: (A) Medicamento: sistema alimentado com a lista de todos os nomes de medicamentos pela DCB padronizados na instituição disponíveis para prescrição ambulatorial. (B) Dose: número de comprimidos/cápsulas/mililitros/mg prescritas. (C) Unidade - especificação do medicamento: unidades para dispensação dos medicamentos cadastrados. (D) Frequência: forma de administração do medicamento com opções pré-preenchidas (ex: 8/8 horas, 1 vez ao dia, a cada 7 dias.). (E) Via de administração: via pela qual a administração do medicamento será realizada. (F) Duração do tratamento: período no qual o medicamento será utilizado. (G) Validade da receita: após o preenchimento do campo duração do tratamento o campo validade é preenchido automaticamente.

Fonte: Rezende LHO, et al., 2018.

Com a implantação de sistemas de prescrições eletrônicas voltados para o atendimento de pacientes ambulatoriais, com um formato mais elaborado de acordo com as necessidades inerentes a este tipo de atendimento e com as informações necessárias para uma prescrição mais correta e clara, a presença de não 
conformidades nas prescrições pode ser em sua grande maioria, eliminada. Vale ressaltar também que é preciso conscientizar os prescritores quanto à importância dos programas de treinamento para utilização do sistema, que visa limitar os erros de prescrições mal elaboradas (CASSIANI SHB, et al., 2003).

Assim, sugere-se como bastante válida a oferta de palestras e de materiais informativos de fácil acesso, como folders, cartazes e cartilhas, tanto aos profissionais como também à população, para conscientizá-la quanto ao seu direito de receber uma prescrição médica adequada, que é amparado por legislações específicas e que traga o correto entendimento das informações ali prestadas evitando com isso erros de medicação. Através de estratégias que visem um melhor aperfeiçoamento por parte daqueles que prescrevem, será possível tornar as prescrições mais detalhadas e fáceis de serem compreendidas pelos profissionais que as manuseiam, bem como para os pacientes que as utilizam para obter informações sobre como administrar os medicamentos ali prescritos.

\section{CONCLUSÃO}

Os resultados obtidos pelo presente estudo demostram que em ambas as formas de elaboração de receitas, manuscritas ou digitadas, tanto os erros de redação, quanto os de decisão foram encontrados. Tais resultados subsidiam a importância da implantação de um modulo de prescrição eletrônica, que promoveria não somente a eliminação da ilegibilidade e a redução do uso de abreviações inadequadas, mas também a não utilização da $\mathrm{DCB}$, denominados erros de redação. Poderia também garantir a correção dos erros de decisão, oriundos da ausência de informações importantes, inerentes aos esquemas farmacoterapêuticos prescritos para o paciente.

\section{REFERÊNCIAS}

1. ALVES TNP, et al. Análise das prescrições médicas em Unidades de Atenção Primária à Saúde do município de Juiz de Fora-MG. Convibra, n. February 2012, p. 17, 2013.

2. ARAUJO PT-DE-B, UCHOA SAC. Avaliação da qualidade da prescrição de medicamentos de um hospital de ensino. Ciênc. saúde coletiva, Rio de Janeiro, 2011, 16(supl. 1): 1107-1114.

3. BRASIL. Decreto $n^{\circ} 20.931$ de 11 de janeiro de 1932, revigorado pelo Decreto de 12 de julho de 1991. Regula e fiscaliza o exercício da medicina, da odontologia, da medicina veterinária e das profissões de farmacêutico, parteira e enfermeira, no Brasil, e estabelece penas. Disponível em: http://www.planalto.gov.br/ccivil_03/decreto/19301949/D20931.htm. Acesso em: 30 set. 2019.

4. BRASIL, Lei 5.991, de 17 de dezembro de 1973.Dispõesobre o Controle Sanitário do Comércio de Drogas, Medicamentos, Insumos Farmacêuticos e Correlatos, e dáoutras Providências. Disponível em http://www.jusbrasil.com.br/legislacao/110058/lei-5991-73. Acesso em: 28 mar. 2018.

5. BRASIL. Lei Federal 9.787, de 10 de fevereiro de 1999. Altera a Lei no 6.360, de 23 de setembro de 1976, que dispõe sobre a vigilância sanitária, estabelece o medicamento genérico, dispõe sobre a utilização de nomes genéricos em produtos farmacêuticos e dá outrasprovidências. Diário Oficial da União 1999.Disponível em: URL http://elegis.bvs.br/leisref/public/ search.php. Acesso em: 30 mar. 2018.

6. BRASIL. Portaria 1554, de 30 de julho de 2013. Dispõe sobre as regras de financiamento e execução do Componente Especializado da Assistência Farmacêutica no âmbito do Sistema Único de Saúde (SUS). Diário Oficial da União. 2013. Disponível em: URL http://portalarquivos.saude.gov.br/images/pdf/2014/abril/02/pt-gm-ms-1554-2013-alterada1996-2013.pdf. Acesso em: 30 abr. 2018

7. BRASIL. Portaria SNVS/MS no. 344, de 12 de maio de 1998. Aprova o RegulamentoTécnico sobre substâncias e medicamentos sujeitos a controle especial. Republicada no DiárioOficial da União. 1999. Disponível em: http://portal.anvisa.gov.br/documents/10181/2718376/PRT_SVS_344_1998_COMP.pdf/a3ee82d3-315c-43b1-87cfc812ba856144. Acesso em: 4 jun. 2018.

8. CARVALHO VT, et al. Erros mais comuns e fatores de risco na administração de medicamentos em unidades básicas de saúde. Ver. LatinoamEnferm. 1999; 7:67-75.

9. CASSIANI SHB, et al. Computerized physician order entry in a university hospital: writing failureand user's opinions. RevEscEnferm USP 2003; 37(4): 51-60.

10. CONSELHO FEDERAL DE FARMÁCIA (Brasil). Resolução 357, de 20 de abril de 2001. Aprova o regulamento técnico das boas práticas de farmácia. Diário Oficial [da República Federativa do Brasil], Poder Executivo, Brasília, 27 abr. 2001. p. 24-31. Disponível em: http://www.cff.org.br/userfiles/file/resolucoes/357.pdf. Acesso em: 20 mai. 2018.

11. CONSELHO FEDERAL DE MEDICINA (Brasil). Resolução CFM nำ1.885/2008. Dispõe sobre o Código de Ética Médica. Brasília: CFM, 1988. Disponível em:http://www.portalmedico.org.br/resolucoes/CFM/2008/1885_2008.htm. Acesso em: 12 set. 2018.

12. DEAN B, et al. What is prescribing error?Qual Health Care 2000; 9:232-7.

13. EV LS, et al. Avaliação das Prescrições Dispensadas em uma Unidade Básica de Saúde do Município de Ouro Preto, Minas Gerais, Brasil.Latin American JournalofPharmacy, n.27, p.543-547, 2008. 
14. GUZZATO P, BUENO D. Análise de prescrições medicamentosas dispensadas na farmácia de uma Unidade Básica de Saúde de Porto Alegre R.S. Rev HCPA 2007; 27(3).

15. JACOBSEN TF, et al. Análise de erros de prescrição em um hospital da região sul do Brasil. Rev. Bras. Farm. Hosp. Serv. Saúde São Paulo 2015; 6(3): 23-26.

16. LUIZA VL, GONÇALVES CBC. A Prescrição Medicamentosa. In: FUCHS FD e WANNMACHER L. (Eds.). Farmacologia clínica: fundamentos da terapêutica racional. 4. ed. Rio de Janeiro: Guanabara Koogan; 2009.

17. MARINHO JS, et al. Erros redacionais em prescriçõesmédicas em uma policlínica de Manaus, Amazonas, Brasil. Infarma ciências farmacêuticas. v26:103-110.

18. NERI EDR. Determinação do perfil dos erros de prescrição de medicamentos em um hospital universitário [dissertação]. Fortaleza (CE): Departamento de Farmácia, Faculdade de Farmácia, Odontologia e Enfermagem, Universidade Federal do Ceará; 2004.

19. ORGANIZAÇÃO MUNDIAL DA SAÚDE. Guia para a boa prescrição médica. Porto Alegre: Artmed, 1998.

20. OSORIO-DE-CASTRO, CGS e PEPE, VLE. Prescrição de Medicamentos. Disponível em:<<http://www.ensp.fiocruz.br/portalensp/judicializacao/pdfs/516.pdf >>. Acesso em: 22 de junho de 2018.

21. OTERO MJ, DOMINGUES AG. Acontecimentos adversos por medicamentos: una patologia emergente. Farm. Hosp. $2004 ; 24(4): 258-266$.

22. PEPE VLE, OSORIO-DE-CASTRO CGS.A interação entre prescritores, dispensadores e pacientes: informação compartilhada como possível benefício terapêutico. Cad Saúde Pública, Rio de janeiro, v. 16, n. 3, p. 815-822, 2000.

23. ROSA MB, et al. Legibilidade de prescrições médicas com medicamentos potencialmente perigosos em um hospital de Belo Horizonte, MG. Rev SBRAFH 2003; (2):22-8.

24. ROSA MB, et al. Erros na prescrição hospitalar de medicamentos potencialmente perigosos. Rev. Saúde Pública2009. 6(43): 490-8.

25. SANTOS GEO. Cálculo amostral: calculadora on-line. Disponível em: http://www.calculoamostral.vai.la. Acesso em: 20 set. 2018.

26. SHANE R. Computerized physician order entry: challenges and opportunities. Am J Health SystPharm 2002; 59:28688.

27. WHO World Health Organization. World alliance for patient safety: forward programme 2006-2007. Geneva; 2006 [citado 2018]. Disponível em: http://www.who. int/patientsafety/em. Acesso em: 05 jun. 2018. 